\title{
Investigation of operational conditions for the removal of methylene blue by Fenton Reaction
}

\author{
Aya Tolpa ${ }^{1}$, Mohamed Gar Alalm ${ }^{2}$, Mohamed Elsamadony ${ }^{1}$, Hafez Afifi ${ }^{1}$
}

\begin{abstract}
Fenton reaction has been concerned by many researchers due to easy operation and effective degradation of bioresistant organics. This study aims to investigate the effect of the operational conditions on the effectiveness of Fenton's process for the decolorization of methylene blue.The influence of methylene blue, $\mathrm{pH}$, dose of $\mathrm{H}_{2} \mathrm{O}_{2}$, ferrous sulfate concentration from the wastewater were studied. Experiments have shown that highly affect by the value of $\mathrm{pH}$, Laboratory experiment conducted in the lab proved that $\mathrm{pH}$ should be between 3 - 4 to give the best results, It also proved that the increasing of the dose of both hydrogen peroxide and ferrous sulfate enhanced the removal efficiency of MB.
\end{abstract}

\section{1- Introduction:}

Dyes are used in many industries that are indispensable in Egypt and the world. They are used in the manufacture of textiles, paper, leather and plastics, which result in severe pollution of industrial wastewater due to the dyes, Scientists have observed the effect of dye toxicity on living organisms [2] , Air, water, land, humanity, plants, animals etc. get affected by industrial pollution. When industrial wastewater is discharged into the waterway, it is hazardous to aquatic life and humans [3]. As that Color is one of the most important criteria required to be controlled, because colored water bodies reduce light penetration, preventing photosynthesis of algae and submerged plants and are aesthetically undesirable [4]. Therefore, it is very difficult to break it biologically. scientists looked for an alternative

Advanced oxidation processes are one of the best alternatives to industrial wastewater treatment, which contains non-biodegradable organic pollutants. These factors include the generation of a free hydroxyl root $(\mathrm{OH})$, which degrades most organic pollutants quickly. Fenton is a well-studied AOP that uses hydrogen peroxide as an oxidant in the presence of a catalyst. Interaction in the advanced oxidation processes As follow, the organic pollutants of the industrial waste water react with hydrogen peroxide in the presence of iron salts such as ferrous sulfate on acidic and better within the limits 3-4 The resulting hydroxyl radicals resulting from the interaction between iron salt (II) and $\mathrm{H}_{2} \mathrm{O}_{2}$ attack the organic aggregates methylene blue as the unsaturated pigment molecule.
Therefore, both the chromophore and the chromogen of the dye molecules will be destroyed and their color removed[1] . 2. Materials and methods:

\section{Material}

Dye, $\mathrm{FeSO}_{4} .7 \mathrm{H}_{2} \mathrm{O}$, and $\mathrm{H}_{2} \mathrm{O}_{2}$ were purchased from $\mathrm{El}$ Gomhouria company. Dye solution of methylene blue was prepared with distilled deionized water. Ferrous sulfate was prepared with distilled deionized water with concentration $(5 \mathrm{gm} / \mathrm{l})$. Analytical grade ferrous sulfate heptahydrate $\left(\mathrm{FeSO}_{4} .7 \mathrm{H}_{2} \mathrm{O}\right)$ and medical extra pure grade $(35 \% \mathrm{w} / \mathrm{w})$, hydrogen peroxide $\left(30 \% \mathrm{H}_{2} \mathrm{O}_{2}\right)$, the $\mathrm{PH}$ adjustment and were performed with HCL to decrease $\mathrm{pH}, \mathrm{NaOH}$ to increase $\mathrm{pH}$.

\section{Method}

Experiments were carried out at room temperature, with 100 $\mathrm{mL}$ volume samples. The $\mathrm{pH}$ of the samples was adjusted to 3 \pm 0.1 using HCL before oxidation. Under rapid mixing conditions $(300 \mathrm{rpm})$ proper volumes of ferrous sulfate and hydrogen peroxide solutions were added to the reactor, respectively, Following the rapid mixing, the samples were stirred by a magnetic stirrer at for 70 minutes at $300 \mathrm{rpm}$, The solids were allowed to settle for 10 minutes and It was centrifuged until all sediment was attached to the pipe used in the expulsion and the sample was taken for analysis. The absorbance measurements by spectrophotometer so that the wavelength of the dye used is adjusted at 660 , the device is calibrated with distilled water then we take readings for each sample.

\section{Absorbance analysis:}

Spectrophotometer (American German laboratory) is used to measure the concentration of dye. the light absorption of a substance is selective as a substance is excited by the radiation of the light, the light absorption effect will occur in the substance. each substance has their own special absorption spectrum. when a homogenous light passes through a solution, the light energy will be lessened, because some of the energy is absorbed by the solution. the loss of energy is in proportion to the concentration of the substance. the series of instruments can perform chemical quantitative analysis on a sample substance according to the principle of colorimetry. within the

Department of public works engineering, faculty of Engineering, Tanta university ${ }^{1}$ Department of public works engineering, faculty of Engineering, Mansoura university ${ }^{2}$ 
range of concentration, all parameter are in compliance with the Beers the range of concentration, all parameter are in compliance with the Beers low:

$\mathrm{A}=\mathrm{I} \mathrm{g}=\mathrm{I} / \mathrm{T}=\mathrm{KCL} \quad, \mathrm{T}=1 / \mathrm{Io}$

\section{Fenton's oxidation studies}

Experiments were conducted on $\mathrm{pH}$ values ranging from 2 to 6 to find out the effect of $\mathrm{pH}$ on the removal efficiencies of color laboratory experiments conducted in the lab proved that maximum color removal efficiencies for dye solutions are obtained at $\mathrm{pH}=3.0$ and, When $\mathrm{pH}$ is increased from 4 to 6 , the removal efficiency is reduced . Figure(1). These results show that increasing the dose of hydrogen peroxide enhanced the removal of color dye solutions. It appears that hydrogen peroxide plays a crucial role in the Fenton's oxidation process in the presence of sufficient ferrous ion under acidic conditions. Decolorization was also affected by temperature. The results show that the proper temperature was $40^{\circ} \mathrm{C}$ for the decolorization of methylene blue dye solution Figure (5). However, this effect was very limited and the change of removal efficiency between the temperatures studied was $1 \%$. Therefore, the following experiments were performed at room temperature.

\section{3 - Results and discussion}

Effect of the Initial methylene blue

Concentration:

As shown in Fig. 1, it is important to investigate how the concentration of initial dye influences color removal. Thus, Stock of methylene blue dye was $1 \mathrm{~g} / \mathrm{L}$ and was replaced with distilled water. Experiments were conducted at different concentrations $(20,25,40,50 \mathrm{mg} / \mathrm{l})$.

By increasing the dye concentration in the Fenton process, many intermediate compounds arose after the degradation of the basic dye that could interfere with the oxidation process. Such repression will be more pronounced in the presence of a large amount of intermediate decomposition products that occurred through increased dye concentration [5].

From the above, we note that the concentration of the dye of methylene blue and the ratio of ejaculation are inversely proportional. The less dye concentration used, the higher the percentage of removal .

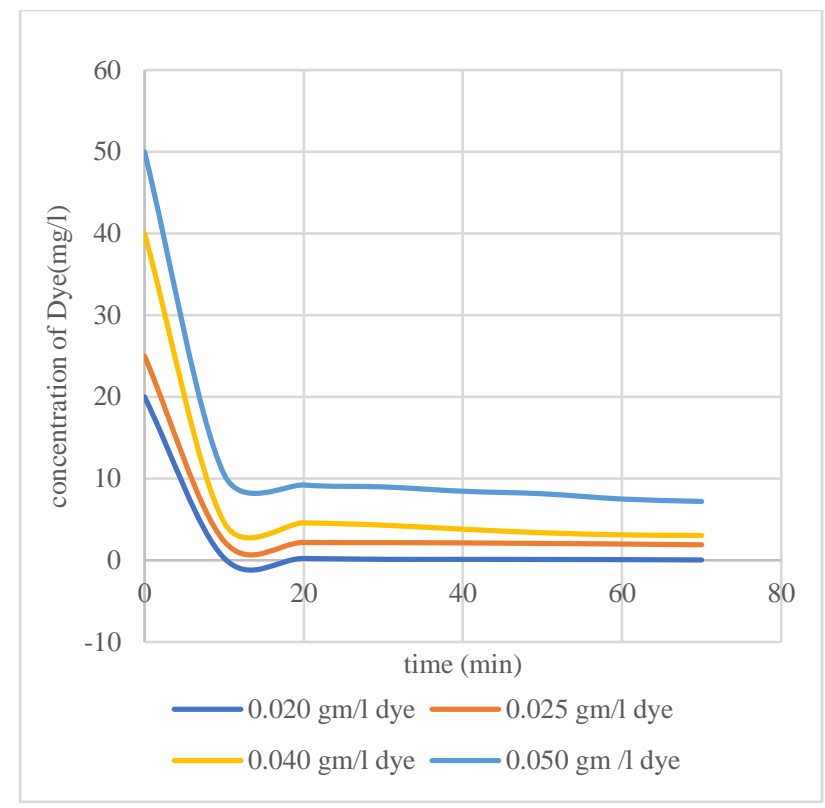

Figure (1). Effect of methylene blue concentration on degradtion methylene blue by time by time : Reaction time $=70 \mathrm{~min}$.

\section{Effect of pH on degradation of methylene blue :}

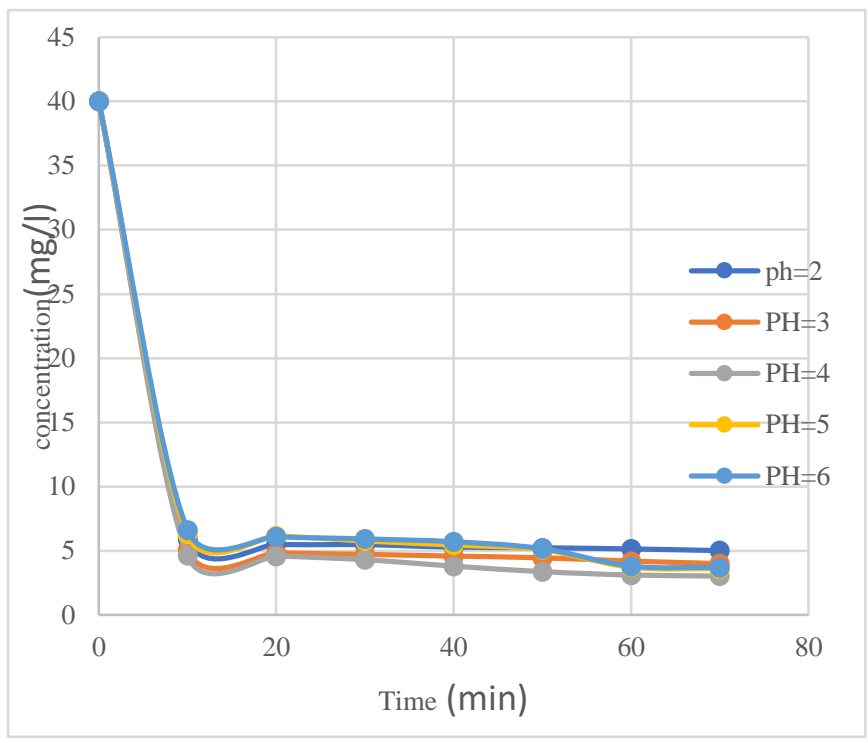

Fig(2) Effect of $\mathrm{pH}$ on dye degradation by time ; Reaction time $=70 \mathrm{~min}$

In spite of the significant effect that the value of $\mathrm{pH}$ has on the results of the reaction, it can't alone, achieve the best results. It also affects the concentration of dye and concentration of hydrogen peroxide to find the optimal value for $\mathrm{pH}$ the experiments were carried out at different $\mathrm{pH}$ range is from $2-6$ by adding $\mathrm{HCl}$ or $\mathrm{NaOH}$ [6] .

Experiments have shown that the optimal value is given the best results at $\mathrm{pH} 3-4$ [7]. AS shown in fig(2). When the $\mathrm{pH}$ $=3-4$ are formed more $\mathrm{Fe}(\mathrm{OH})$ and be more active than $\mathrm{Fe} 2$ this may be the reason for the disintegration of the methylene 
blue [8].

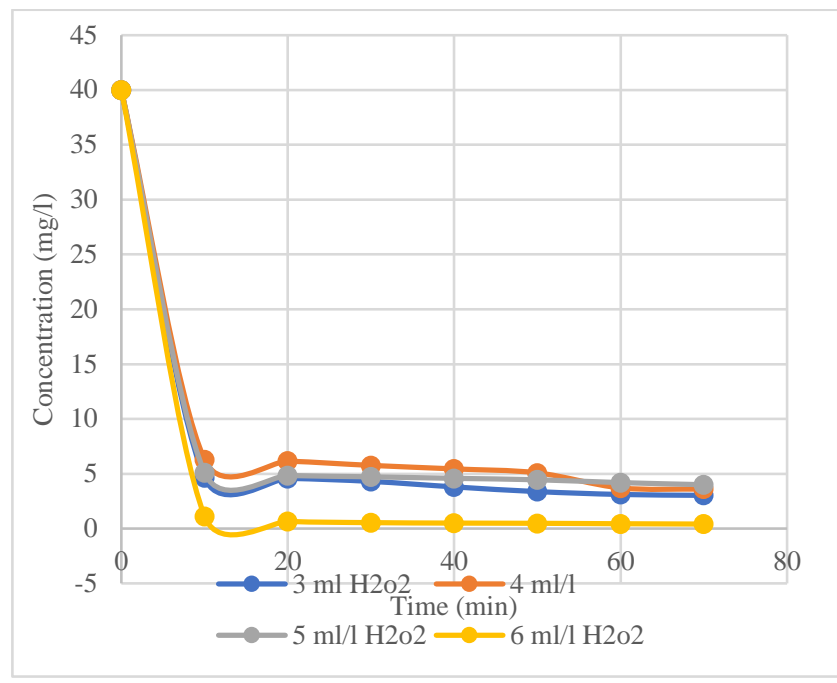

Figure (3) Effect of $\mathrm{H}_{2} \mathrm{O}_{2}$ concentration on degradation of methylene blue by time; : Reaction time $=70 \mathrm{~min}$

\section{The variation of Hydrogen peroxide during the Fenton reaction :}

Increasing the concentration of hydrogen peroxide to accelerate the clearance of methylene blue at the beginning of the reaction, while increasing the concentration of hydrogen peroxide during the reaction, the efficiency of removal decreased. This could be because the hydroxyl radicals generated produce hydroperoxyl radicals (HO2 $\bullet$ ) in the presence of a local excess of $\mathrm{H} 2 \mathrm{O} 2$. The hydroperoxyl radicals are much, less reactive and do not contribute to the oxidative degradation of the organic substrate which takes place only through the reaction with $\mathrm{HO} \cdot[9]$.

\section{Effect of hydrogen peroxide dose on methylene blue degradation:}

Hydrogen peroxide is used in advanced processes (APs), as hydroxyl radicals source, is the best-known AP utilizing $\mathrm{H} 2 \mathrm{O} 2$ with ferrous/ferric ion at low $\mathrm{pH}$, during which hydrogen radicals are generated. When concentration of hydrogen peroxide increased, the $\mathrm{pH}$ decreased [10]. As shown in fig. 3 , the optimum dose of hydrogen peroxide is 6 $\mathrm{ml} / 1$, achieved percentage removal $89.9 \%$

\section{Effect of FeSO4.7H2O concentration on methylene blue degradation:}

The rate of degradation is dramatically increased when the ferrous sulfate concentration is increased A solution of the 1 ferrous sulfate was treated as a stock at a concentration of $5 \mathrm{~g}$ / $\mathrm{L}$ and used concentrations at range $(0.1 \mathrm{gm} / 1: 1 \mathrm{gm} / \mathrm{l})$. As shown in fig. 4 .Therefore, increasing the concentration of FeSO $4 \cdot 7 \mathrm{H} 2 \mathrm{O}$ has stronger effect on $\mathrm{MB}$ degradation than increasing the concentration of hydrogen peroxide. Ferrous sulfate interacts with hydrogen peroxide to generate hydroxyl radicals which enhance degrades the dye according to the following reaction[11].

$$
\mathrm{Fe} 2++\mathrm{H} 2 \mathrm{O} 2 \rightarrow \mathrm{Fe} 3++\mathrm{OH} \bullet+\mathrm{OH}^{-}(1)
$$

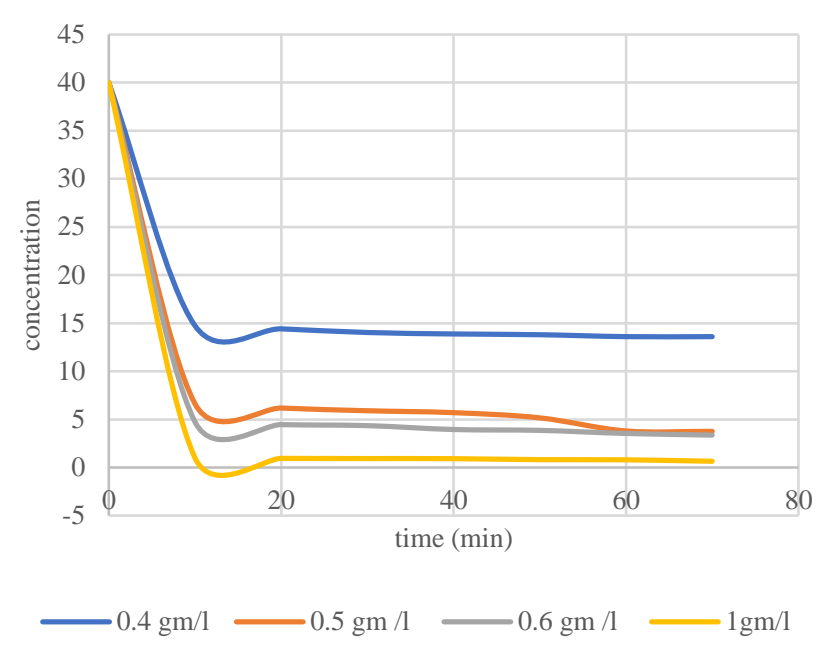

Figure (4) Effect of concentration of ferrous sulfate degradation of methylene blue by time $;$ Reaction time $=70 \mathrm{~min}$

Effect of temperature on methylene blue degradation: The effect of temperature at room temperature, $30^{\circ} \mathrm{c}$ and $40^{\circ}$ $\mathrm{c}$ on $\mathrm{MB}$ degradation was investigated. It can be seen from Fig. 5 that increasing temperature had a positive effect on the MB degradation [12] .

Effect of interaction time on methylene blue degradation:

As shown in Fig(6) time enhanced the decomposition of methylene blue, MB was found to be almost decomposed at all in 30 minutes, if only hydrogen peroxide or iron ions were added. Therefore, it can be confirmed from the figure that MB is heavily oxidized by the Fenton detector. The complete decomposition. It is possible to say that the reaction time can be divided into two phases. Interaction in the first minute of the reaction is the fastest reaction. Approximately $70 \%$ of the pigmentation can occur at the first minute [8]. The second part of the decomposition of the dye is slow taking about 30 minutes to deteriorate approximately $97 \%$. The main reason for the reaction in two phases is hydrogen. The phase name $\mathrm{Fe}$ $2+/ \mathrm{H} 2 \mathrm{O} 2$. Ferric ions can react with hydrogen peroxide to detect hydroxyl $(\mathrm{HO} 2+)$ iron wafers, and iron ions react with hydrogen peroxide to produce hydroxyl radicals, Hydroxyl roots are formed [8]. 
Vol. 1 - 2019

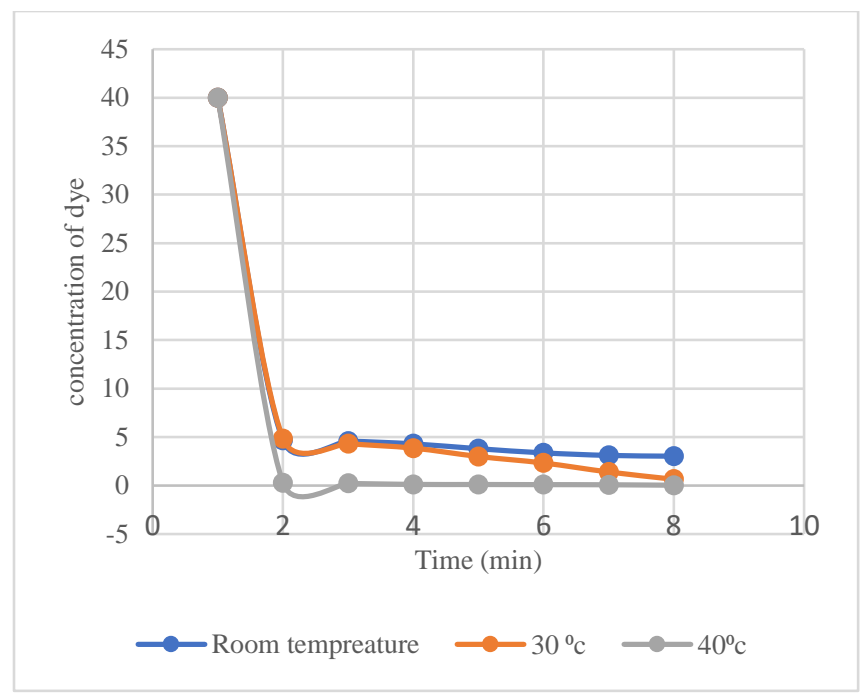

Figure (5) Effect of temperature on degradation of methylene blue by time ;Reaction time $=70 \mathrm{~min}$

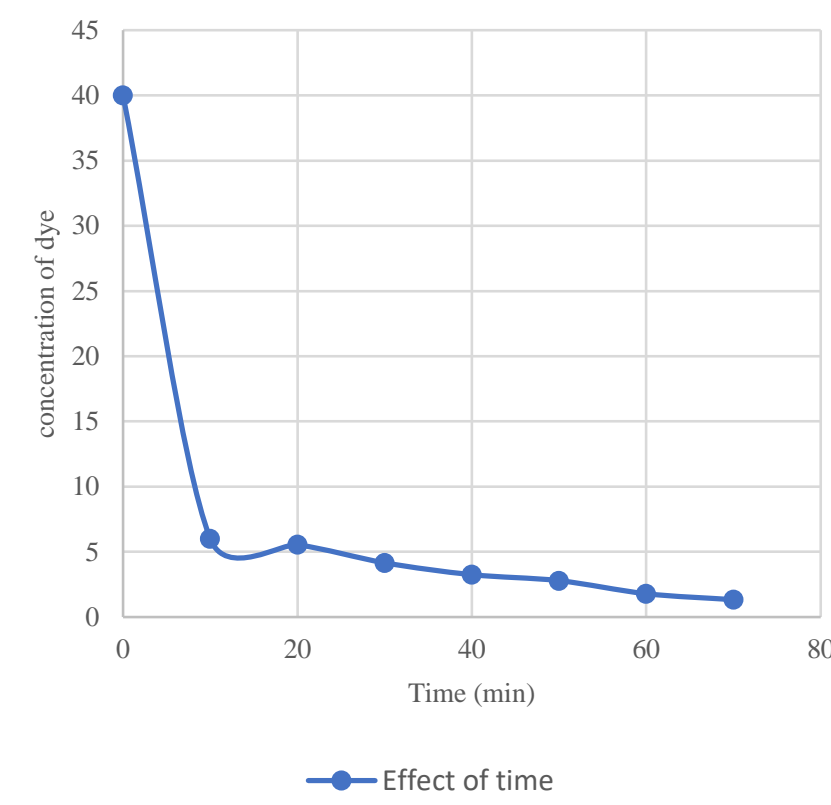

Figure (6) Effect of Time on degradation of methylene blue by time ; Reaction time $=70 \mathrm{~min}$

\section{Conclusion}

From the results of this study.

- The degradation of MB efficiency by oxidation was greatly affected by the $\mathrm{pH}$ reaction. The most effective $\mathrm{pH}$ range was observed at 4.0 and below. The degradation of $\mathrm{MB}$ it is also affected by the dose of hydrogen peroxide. The most effective $\mathrm{H} 2 \mathrm{O} 2$ dose is $6 \mathrm{ml} / \mathrm{l}$.

- The highest color removal efficiency of $99.85 \%$ was achieved for an external addition of $60 \mathrm{ml} / \mathrm{L}$ hydrogen peroxide with $1.0 \mathrm{~g} / \mathrm{L}$ methylene blue and $0.5 \mathrm{~g} / \mathrm{L}$ Ferrous sulfate at $40^{\circ} \mathrm{c}, \mathrm{pH} 4$ after $70 \mathrm{~min}$.
Engineering Research Journal

- Without temperature effect, the highest color removal efficiency of $98.33 \%$ was achieved for an external addition of $6 \mathrm{ml} / \mathrm{L}$ hydrogen peroxide with $1.0 \mathrm{~g} / \mathrm{L}$ methylene blue and $0.5 \mathrm{~g} / \mathrm{L}$ Ferrous sulfate, $\mathrm{pH} 4$ after $70 \mathrm{~min}$. From laboratory experiments, we can determine the optimal dose that gave the best removal rate $5 \mathrm{gm} / \mathrm{l}$ ferrous sulfate, 6 $\mathrm{ml} / \mathrm{l} \mathrm{H} 2 \mathrm{o} 2$.

\section{Reference}

[1] M. F. Sevimli and C. Kinaci, "Decolorization of textile wastewater by ozonation and Fenton's process," no. August, pp. 279-286, 2018.

[2] V. M. Correia, T. Stephenson, and S. J. Judd, "Environmental Technology Characterisation of textile wastewaters-a review CHARACTERISATION OF TEXTILE WASTEWATERS-A REVIEW," Environ. Technol., pp. 917-929, 1994.

[3] J. Khatri, P. V Nidheesh, T. S. A. Singh, and M. S. Kumar, "Advanced oxidation processes based on zero-valent aluminium for treating textile wastewater," Chem. Eng. J., vol. 348, no. April, pp. 67-73, 2018 .

[4] C. S. D. Rodrigues, L. M. Madeira, and R. A. R. Boaventura, "Treatment of textile dye wastewaters using ferrous sulphate in a chemical coagulation/flocculation process," Environ. Technol. (United Kingdom), vol. 34, no. 6, pp. 719-729, 2013.

[5] J. Chalfin and A. G. Urkowitz, "Model of Coal Conversion and Its Effect on Power Generating Costs and Air Quality.," Coal Technol. Int. Coal Util. Conf. Exhib., pp. 345-357, 1983.

[6] K. Dutta, S. Mukhopadhyay, S. Bhattacharjee, and B. Chaudhuri, "Chemical oxidation of methylene blue using a Fenton-like reaction," J. Hazard. Mater., vol. 84, no. 1, pp. 57-71, 2001.

[7] W. G. Kuo, "DECOLORIZING DYE WASTEWATER WITH FENTON'S REAGENT,” vol. 26, no. 7, pp. 881-886, 1992.

[8] P. K. Malik and S. K. Saha, "Oxidation of direct dyes with hydrogen peroxide using ferrous ion as catalyst," vol. 31, pp. 241-250, 2003.

[9] M. Neamţu, C. Catrinescu, and A. Kettrup, "Effect of dealumination of iron(III) - Exchanged Y zeolites on oxidation of Reactive Yellow 84 azo dye in the presence of hydrogen peroxide," Appl. Catal. B Environ., vol. 51, no. 3, pp. 149-157, 2004.

[10] O. Silverman-Retana, E. Servan-Mori, R. Lopez-Ridaura, and S. Bautista-Arredondo, "Diabetes and hypertension care among male prisoners in Mexico City: exploring transition of care and the equivalence principle," Int. J. Public Health, vol. 61, no. 6, pp. 651$659,2016$.

[11] S. Liu, J. Huang, Y. Ye, A. Zhang, L. Pan, and X. Chen, "Microwave enhanced Fenton process for the removal of methylene blue from aqueous solution," Chem. Eng. J., vol. 215-216, pp. 586-590, 2013.

[12] X. R. Xu and X. Z. Li, "Degradation of azo dye Orange G in aqueous solutions by persulfate with ferrous ion," Sep. Purif. Technol., vol. 72, no. 1, pp. 105-111, 2010. 